\title{
SPECTRALLY TWO-UNIFORM FRAMES FOR ERASURES
}

\author{
Saliha Pehlivan, Deguang Han and Ram Mohapatra
}

\begin{abstract}
We continue to work on the problem of characterizing erasure-optimal frames when spectral radius is used as a measurement of the error operator. Spectrally optimal $(N, n)$-frames for one erasures are the ones that the minimal spectral error $n / N$ can be achieved. This class of frames was completely characterized in [28] in terms of the connectivity property and the redundancy distributions of the involved frames. We show that the best spectral error for the two erasures is always greater than or equal to $\frac{n}{N}+\left(\frac{N n-n^{2}}{N^{2}(N-1)}\right)^{1 / 2}$. We characterize all the frames such that the above lower bound can be achieved. Different characterizations are also obtained for the case that when $N=n+1$ or $n+2$. We show that in these special cases, spectrally 2 -erasure optimal frames are related to the $n$-independence property of frames.
\end{abstract}

Mathematics subject classification (2010): Primary 42C15, 46C05, 47B10.

Keywords and phrases: Frames, erasures, spectrally optimal dual frames, connected frames, redundancy distribution, $k$-independent sets.

\section{REFERENCES}

[1] J. Benedetto And M. Fickus, Finite normalized tight frames, Adv. Comput. Math., 18 (2003), 357-385.

[2] B. Bodmann, Optimal linear transmission by loss-insensitive packet encoding, Appl. Comput. Harmon. Anal., 22 (2007), 274-285.

[3] B. Bodmann And V. I. Paulsen, Frames, graphs and erasures, Linear Algebra Appl. 404 (2005), $118-146$.

[4] B. Bodmann, V. Paulsen and M. Tomforde, Equiangular tight frames from complex Seidel matrices containing cube roots of unity, Linear Algebra Appl., 430 (2009), 396-417.

[5] B. Bodmann AND P. Singh, Burst erasures and the mean-square error for cyclic Parseval frames, IEEE Trans. Inform. Theory, 57 (2011), 4622-4635.

[6] J. Cahill, M. Fickus, D. G. Mixon, M. J. Poteet and N. K. Strawn, Constructing finite frames of a given spectrum and set of lengths, Appl. Comput. Harm. Anal., 35 (2013), 52-73.

[7] P. Casazza, A. Heinecke, F. Krahmer, G. Kutyniok, Optimally Sparse Frames, IEEE Trans. Inform. Theory, 57 (2011), 7279-7287.

[8] P. Casazza And J. Kovavcević, Equal-norm tight frames with erasures, Adv. Compt. Math., 18 (2003), 387-430.

[9] O. Christensen, A. M. Powell and X. C. Xiao, A note on finite dual frame pairs, Proc. Amer. Math. Soc., 140 (2012), 3921-3930.

[10] D. M. Duncana, T. R. Hoffman, J. P. Solazzo, Equiangular tight Frames and fourth root Seidel matrices, Linear Algebra and its Applications 432 (2010), 2816-2823.

[11] D. DutKay, D. HAN AND D. LARSON, A duality principle for groups, J. Funct. Anal., 257 (2009), $1133-1143$.

[12] Y. Eldar And H. BölcskeI, Geometrically uniform frames, IEEE Trans. Info. Theory, 49 (2003), 993-10006.

[13] M. Fickus, D. G. Mixon, M. J. Poteet, N. Strawn, Constructing all self-adjoint matrices with prescribed spectrum and diagonal, Advances in Computational Mathematics 39 (2013), 585-609.

[14] V. K. Goyal, J. Kovavcević, And J. A. Kelner, Quantized frame expansions with erasures, Appl. Comput. Harmon. Anal. 10 (2001), 203-233. 
[15] D. Han, K. Kornelson, D. LARson, AND E. Weber, Frames for Undergraduates, volume 40 of Student Mathematical Library, American Mathematical Society, 2007.

[16] D. Han And D. R. Larson, Frames, Bases and Group Representations, Mem. Amer. Math. Soc., 147 (697): 1-94, 2000.

[17] D. Han, D. Larson, B. LiU And R. Liu, Operator-Valued Measures, Dilations, and the Theory of Frames, Mem. Amer. Math. Soc., Vol. 229, No. 1075 (2014).

[18] T. Hoffman, J. Solazzo, Complex equiangular tight frames and erasures, Linear Algebra and its Applications, 437 (2012), 54-558.

[19] R. Holmes, V. Paulsen, Optimal frames for erasures, Linear Algebra and its Applications, 377 (2004), 31-51.

[20] D. KalRA, Complex equiangular cyclic frames and erasures, Linear Algebra and its Applications, 419 (2006), 373-399.

[21] J. Kovavcević And A. CheBira, Life beyond bases: The advent of frames, IEEE Signal Proc. Mag., 2007.

[22] F. Krahmer, G. Kutyniok, And J. Lemvig, Sparsity and spectral properties of dual frames, Linear Algebra and its Applications, 439 (2013), 982-998.

[23] J. LENG, D. HAN, Optimal dual frames for erasures, Linear Algebra and its Applications, 435 (2011), $1464-1472$.

[24] J. LenG, D. HAN AND T. HuAng, Optimal dual frames for communication coding with probabilistic erasures, IEEE Trans. Signal Proceccing, 59 (2011), 5380-5389.

[25] J. Lopez, D. HAN, Optimal dual frames for erasures, Linear Algebra and its Applications, 432 (2010), 471-482.

[26] P. MASSEY, M. RuIZ AND D. STOJANOFF, Optimal dual frames and frame completions for majorization, Appl. Comput. Harmon. Anal., 34 (2013), 201-223.

[27] P. Massey, M. Ruiz, D. Stojanoff, Optimal completions of a frame, submitted, Adv. Comput. Math., 40 (2014), 1011-1042.

[28] S. Pehlivan, D. Han and R. Mohapatra, Linearly Connected Sequences and Spectrally Optimal Dual Frames for Erasures, Journal of Functional Analysis, 265 (2013) 2855-2876.

[29] T. STROHMER AND R. W. HEATH, Grassmannian frames with applications to coding and communication, Appl. Comp. Harmonic Anal., 14 (2003), 257-275. 